\title{
miRNA-148a inhibits cell growth of papillary thyroid cancer through STAT3 and PI3K/AKT signaling pathways
}

\author{
YUAN XU, YI-FAN HAN, SHAO-JUN ZHU, JIAN-DA DONG and BING YE \\ Department of General Surgery, The Second Affiliated Hospital of \\ Wenzhou Medical University, Wenzhou, Zhejiang 325000, P.R. China
}

Received March 13, 2017; Accepted August 7, 2017

DOI: $10.3892 /$ or.2017.5947

\begin{abstract}
The function of miRNA-148a in lymphatic metastases of papillary thyroid cancer and its mechanism were tested. In this investigation, miRNA-148a expression of lymphatic metastases of papillary thyroid cancer patients was inhibited, compared with normal group. We found that miRNA-148a overexpression was effectively reduced cell cell proliferation and metastases, and induced apoptosis of papillary thyroid cancer in vitro. Overexpression of miRNA-148a significantly induced Bax protein expression and caspase-3/9 levels, and suppressed phosphorylation STAT3 (p-STAT3), PI3K and p-Akt protein expression of papillary thyroid cancer in vitro. Next, si-STAT3, could inhibit p-STAT3 protein expression, reducing cell-cell proliferation and metastases, and inducing apoptosis of papillary thyroid cancer following miRNA-148a overexpression. Then, the PI3K inhibitor was able to inhibit PI3K and p-Akt protein expression, reduced cell cell proliferation and metastases, and induced apoptosis of papillary thyroid cancer following miRNA-148a overexpression. Taken together, our results suggest that miRNA-148a inhibits lymphatic metastases of papillary thyroid cancer through STAT3 and PI3K/AKT signaling pathways.
\end{abstract}

\section{Introduction}

Papillary thyroid cancer (PTC) ranks the first among endocrine tumors and head and neck tumors. According to statistics in recent years, the morbidity of PTC shows an increasing and younger trend, which has become one of the common malignancies (1). In the USA, PTC accounts for 7.5-10\% of all malignancies in population aged 15-24 years (1). Differentiated thyroid cancer (DTC) includes PTC and follicular thyroid

Correspondence to: Dr Yuan Xu, Department of General Surgery, The Second Affiliated Hospital of Wenzhou Medical University, 109 West Xueyuan Road, Wenzhou, Zhejiang 325000, P.R. China E-mail: xfcfcbw88927216@126.com

Key words: miRNA-148a, lymphatic metastases, papillary thyroid cancer, STAT3, PI3K/AKT cancer (FTC), which has the highest morbidity in PTC, accounting for $95 \%$ of all PTCs (2).

The association of miRNA with tumor has been explained gradually accompanied by increase in research on microRNA (miRNA) (3). It is indicated in research that miRNA is closely related to tumor genesis, metastasis, as well as sensitivity of tumor cells to antitumor drugs (4). Therefore, research on miRNA is promising to achieve breakthroughs in terms of non-131I uptake in PTC lung metastasis and loss of differentiation (4).

miRNAs are a class of non-coding single-stranded small RNA molecules with the length of 19-23 nucleotides, which locate in the non-coding region of the genome (3). miRNAs, which are highly conserved in evolution, can regulate gene expression at translation level. Moreover, they can participate in multiple physiological metabolic processes, including development, cell differentiation, cell apoptosis, and cell energy metabolism (5). With the advancement in research on molecular biology and molecular genetics, increasing studies have demonstrated that miRNAs are related to tumor genesis, development, metastasis and prognosis (5). Feng et al showed that microRNA-148a suppressed cell proliferation and migration of pancreatic cancer cells (6). Zhang et al showed that microRNA-148a suppressed cell proliferation and invasion potential through regulation of STAT3 in non-small cell lung carcinomas (7).

Signal transducer and activator of transcription (STAT) is a kind of signal transduction and transcriptional activator, which is also a type of DNA binding protein (8). As the important JAK substrates in the JAK-STAT pathway, STATs play critical roles in the signal transduction of cytokines (CKs). STAT3 gene is a member of the STAT family, which is referred to as the statistical gene (9). Such gene is a part of the basic intracellular chemical signaling pathway, which gives commands to the proteins (9). STAT3 gene has become the hotspot of oncogene research at present since it participates in cell growth and proliferation (10). STAT3 is one of the important members among the signal transcription and activating factors (11). STAT3 has been indicated in substantial research to be closely related to tumor cell proliferation, differentiation, cell apoptosis, angiogenesis, invasion, metastasis, and immune escape (12). Abnormal STAT3 expression and activation has been verified to exist in multiple tumor tissues and cell lines, including malignant melanoma, breast cancer, prostate cancer, 
gastric cancer and colorectal cancer (10). Zhang et al showed that microRNA-148a suppressed cell proliferation and invasion potential through regulation of STAT3 in non-small cell lung carcinomas (7).

Research on the correlation of PI3K/Akt/mTOR signaling pathway with malignant tumor has become the focus in recent years (13). The PI3K/Akt/mTOR signaling pathway not only plays a vital role in the growth and proliferation of normal cells, but is also closely related to the growth of cancer cells. It is the central controller of cell growth and differentiation that is involved in biological processes such as gene transcription, protein translation, and ribosome synthesis; in addition, it plays an extremely important role in cell growth and apoptosis $(13,14)$. Therefore, investigating the correlation of PI3K/Akt/mTOR signaling pathway with PTC can provide a brand-new angle of view for illustrating the pathogenesis and diagnosis of PTC, and probing into the application of its inhibitor can usher in a new path for the targeted therapy of PTC (15). In this study, we tested the function of miRNA-148a in lymphatic metastases of papillary thyroid cancer and its mechanism.

\section{Materials and methods}

Ethics statement and patients. Written informed consent in the study was obtained from all patients before surgery and volunteer at Department of General Surgery, The Second Affiliated Hospital of Wenzhou Medical University. Peripheral blood $(5 \mathrm{ml})$ of patients with lymphatic metastases of papillary thyroid cancer $(n=6)$ and volunteers $(n=6)$ were selected into this study and serum were collected after centrifuge at $2,000 \mathrm{x}$ g for $10 \mathrm{~min}$ and saved at $-80^{\circ} \mathrm{C}$. This study was approved by the ethics committee of The Second Affiliated Hospital of Wenzhou Medical University. Samples were immediately snap-frozen in liquid nitrogen for store.

RNA isolation and $q R T-P C R$. Total RNA was extracted from serum by using TRIzol reagent (Invitrogen, Carlsbad, CA, USA) according to the manufacturer's protocol. Genomic DNA was removed using RNase-free DNase RQ1 (10 U; Promega). Total RNA (100 ng) was used to reverse transcripted cDNA and qRT-PCR was performed with the Bio-Rad Real-Time PCR Detection system (CFX Connect ${ }^{\mathrm{TM}}$; Bio-Rad) using $\mathrm{iQ}^{\mathrm{TM}}$ SYBR $^{\circledR}$ Green Supermix (CFX Connect; Bio-Rad). The relative expression of miRNA-148a was calculated using the $2^{-\Delta C T}$ method.

Cell culture and transfection. Human PTC-derived TPC-1 cells were cultured in RPMI-1640 medium (Gibco, Carlsbad, CA, USA) supplemented with $10 \%$ FBS (Gibco) in a $5 \% \mathrm{CO}_{2}$ atmosphere at $37^{\circ} \mathrm{C}$. miRNA-148a $(50 \mathrm{nM})$, si-STAT3 $(50 \mathrm{nM})$ and negative control mimics $(50 \mathrm{nM})$ were purchased from and transfected into TPC-1 cell using Lipofectamine 3000 (Invitrogen, Guangzhou, China). After transfected with miRNA-148a for $4 \mathrm{~h}, 100 \mathrm{nM}$ of LY294002 was added into cells and cultured for $48 \mathrm{~h}$.

Cell proliferation assay. After transfection for 24,48 and $72 \mathrm{~h}$, cells were placed in 96 -well polystyrene tissue culture plates and $50 \mu 1$ 3-(4,5-dimethylthiazol-2-yl)-2, 5-diphenyltetrazolium

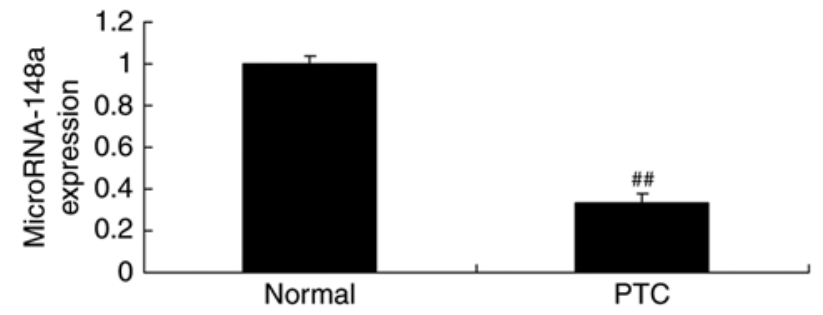

Figure 1. miRNA-148a expression of lymphatic metastases of papillary thyroid cancer patients. Normal, normal group; PTC, papillary thyroid cancer patients group. ${ }^{\# \#} \mathrm{p}<0.01$ compared with normal group.

bromide (MTT, $1 \mu \mathrm{g} / \mathrm{ml}$; Sigma-Aldrich, St. Louis, MO, USA) was added and cultured for $4 \mathrm{~h}$ at $37^{\circ} \mathrm{C}$. Medium was discarded and $150 \mu 1$ dimethylsulfoxide (DMSO; Invitrogen Co., Australia) were loaded in each well for $20 \mathrm{~min}$ at $37^{\circ} \mathrm{C}$. Absorbancy was measured at $492 \mathrm{~nm}$ with Microplate Reader Model 550 (Bio-Rad Laboratories, Japan).

Transwell cell migration assay. After transfection for $24 \mathrm{~h}$, TPC-1 cells were seeded into 24-well Transwell plates (Corning, Corning, NY, USA). After incubation for $24 \mathrm{~h}$, cells were fixed and stained with $2 \%$ crystal violet solution in ethanol (Beyotime Biotechnology). Cell was observed using an IX71 fluorescence microscope (Olympus, Tokyo, Japan).

Assessment of apoptosis. After transfection for $48 \mathrm{~h}$, $1 \times 10^{6}$ cells $/ \mathrm{ml}$ were counted and washed in PBS, re-suspended in binding buffer. Cells were stained with FITC-V and PI (Pharmingen, Becton-Dickinson Co., San Diego, CA, USA), and incubated for $15 \mathrm{~min}$ in the dark at room temperature. Cell apoptosis were analyzed by flow cytometry (FACSCalibur; Becton-Dickinson) using CellQuest software.

Western blot analysis and measurement of caspase-3 activity. After transfection for $48 \mathrm{~h}$, cells were lysed using Radio Immunoprecipitation assay lysis buffer (Beyotime Biotechnology) and protein concentration was determined by using a BCA Protein assay kit (Beyotime Biotechnology). Equal amount of protein was subjected to sodium dodecyl sulfate polyacrylamide gel electrophoresis (SDS-PAGE, 6-10\%), and transferred onto polyvinylidene difluoride (PVDF) membranes (Bio-Rad, Hercules, CA, USA). Western blotting was performed using antibodies against Bax, p-STAT3, PI3K, p-Akt and GAPDH (Cell Signaling Technology) at $4^{\circ} \mathrm{C}$ overnight. Membranes was conducted with a goat anti-rabbit IgG horseradish peroxidase (HRP) conjugated antibody for $1 \mathrm{~h}$ at $37^{\circ} \mathrm{C}$ and visualized using enhanced chemiluminescence (Goodbio Biotechnology). Protein blank was imaged with the Molecular Imager ChemiDoc ${ }^{\mathrm{TM}}$ XRSp (Bio-Rad) and ImageLab software version 4.1 (Bio-Rad). Equal protein was used to measure caspase-3/9 activities using caspase-3/9 activities apoptosis kits (Beyotime Biotechnology). Absorbancy was measured at $405 \mathrm{~nm}$ with Microplate Reader Model 550 (Bio-Rad Laboratories, Japan).

Statistical analysis. Data are presented as the mean \pm SD. Statistical comparisons between two groups were made using one-way ANOVA followed by least significant difference 

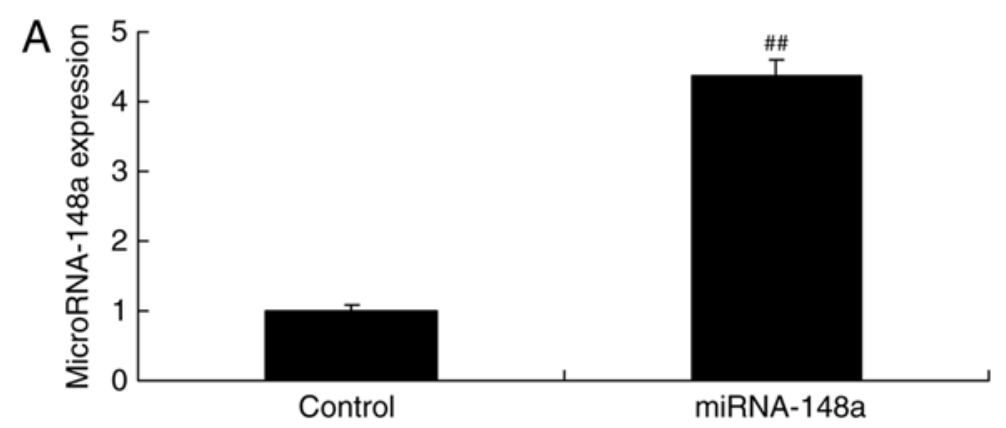

$\mathrm{B}$

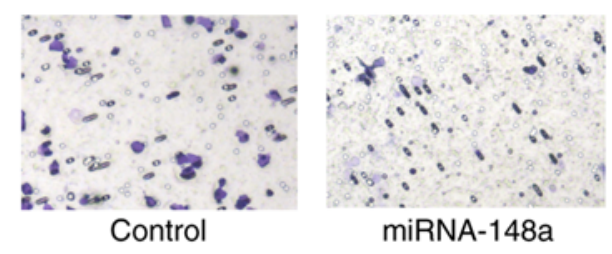

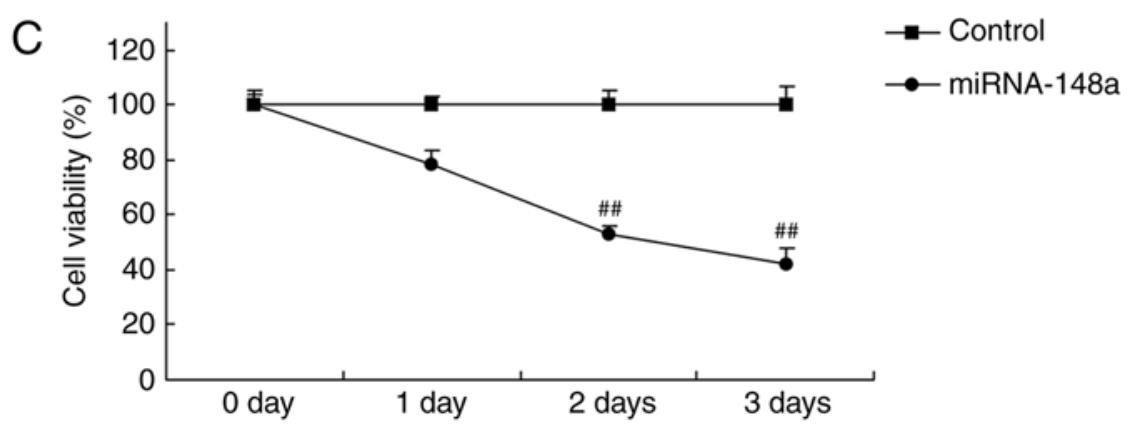

Figure 2. miRNA-148a overexpression reduced cell proliferation and metastases of PTC cells. miRNA-148a overexpression (A), metastases (B) and cell proliferation (C) of PTC cells. Control, control group; miRNA-148a, miRNA-148a overexpression group. ${ }^{\# \#} \mathrm{p}<0.01$ compared with control group.
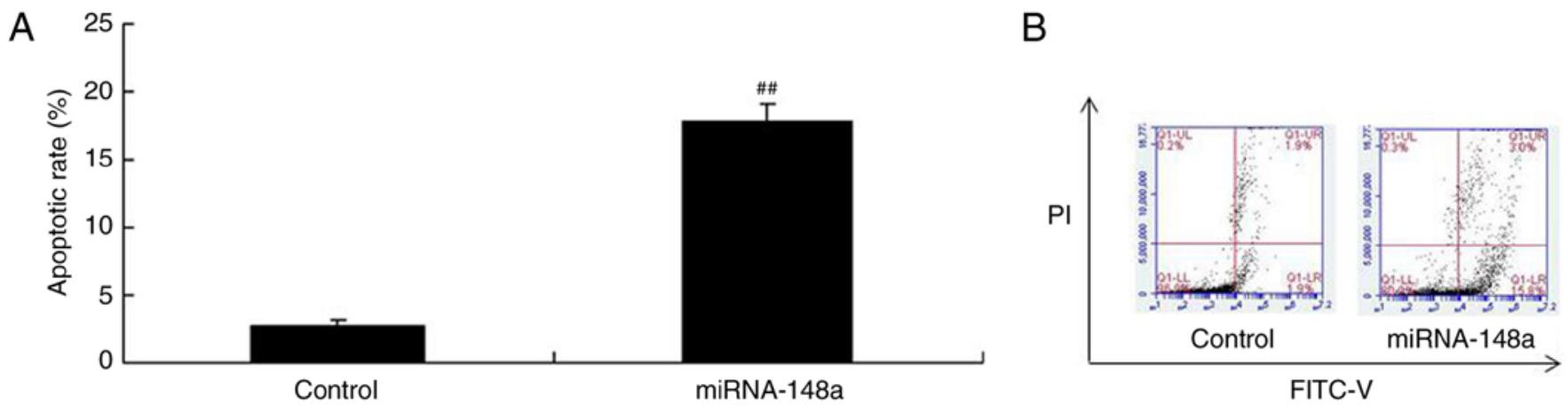

Figure 3. miRNA-148a overexpression reduces apoptosis of PTC cells. miRNA-148a overexpression reduced apoptosis rate (A) and cell apoptosis by flow cytometry (B). Control, control group; miRNA-148a, miRNA-148a overexpression group. ${ }^{\# \#} \mathrm{p}<0.01$ compared with control group.

(LSD) post hoc test. $\mathrm{p}<0.05$ was considered statistically significant.

\section{Results}

miRNA-148a expression of lymphatic metastases of papillary thyroid cancer patients. To explore the role of miRNAs in gastric cancer, we performed qRT-PCR to analyze miRNA-148a expression. As showed in Fig. 1, miRNA-148a expression of lymphatic metastases of papillary thyroid cancer patients was inhibited, compared with normal group.

miRNA-148a overexpression reduces cell cell proliferation and decreased metastases-induced apoptosis of PTC cells. We validated the function of miRNA-148a on cell proliferation and metastases of TC cells. Fig. 2 showed that miRNA-148a mimics increased the expression of miRNA-148a in TPC-1 cells, however, miRNA-148a overexpression reduced cell-cell proliferation and decreased metastases induced apoptosis of PTC cells.

miRNA-148a overexpression induces apoptosis of PTC cell. To verify our hypothesis, we analyzed apoptosis rate in TPC-1 cells by miRNA-148a overexpression using flow cytometry. Conversely, the apoptosis rate of TPC-1 cells transfected with miRNA-148a mimics were higher than that of control group (Fig. 3). These results indicated that miRNA-148a adjusts cell growth and death of PTC, however, its mechanism needs further analysis.

miRNA-148a overexpression induces Bax protein expression and caspase-3/9 levels of PTC cells. Then, we analyzed Bax protein expression and caspase-3/9 levels of PTC cells by miRNA-148a overexpression. We found that miRNA-148a overexpression induced Bax protein expression and caspase-3/9 levels of PTC cells (Fig. 4). 

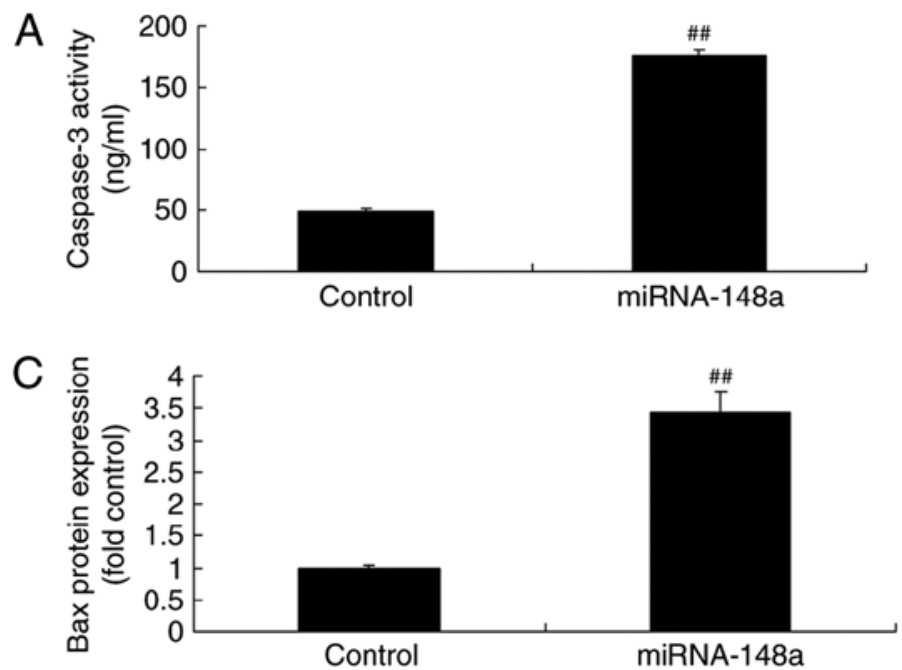
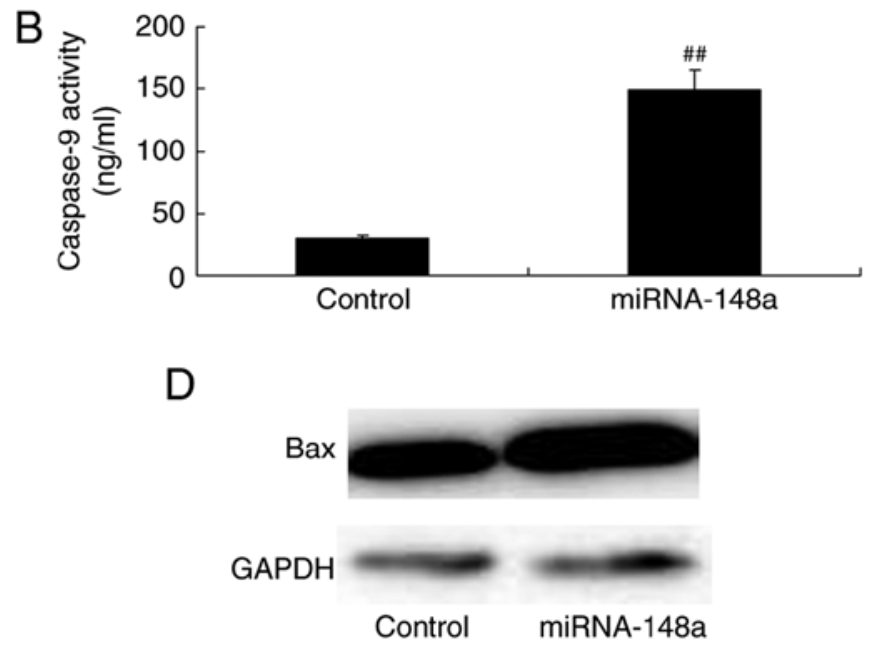

Figure 4. miRNA-148a overexpression induces Bax protein expression and caspase-3/9 levels of PTC cells. miRNA-148a overexpression induced caspase-3/9 levels (A and B), Bax protein expression by statistical analysis (C) and Bax protein expression by western blot analysis (D) of PTC cells. Control, control group; miRNA-148a, miRNA-148a overexpression group. ${ }^{\# \#} \mathrm{p}<0.01$ compared with control group.
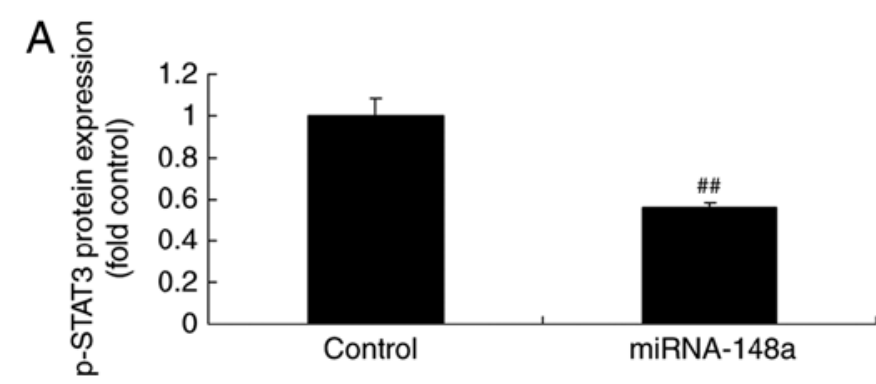

C 흠

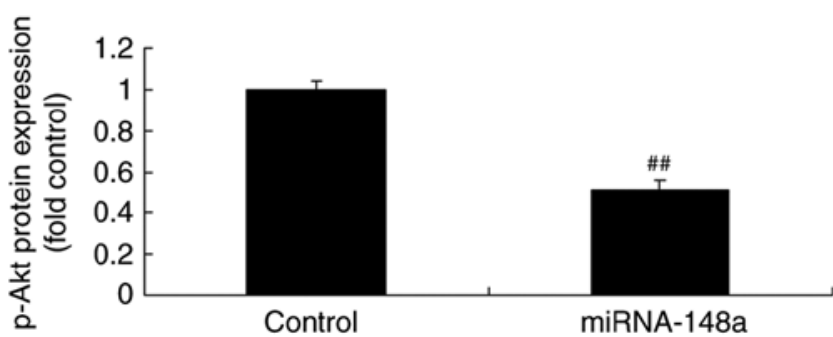

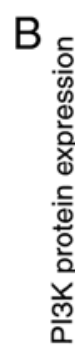
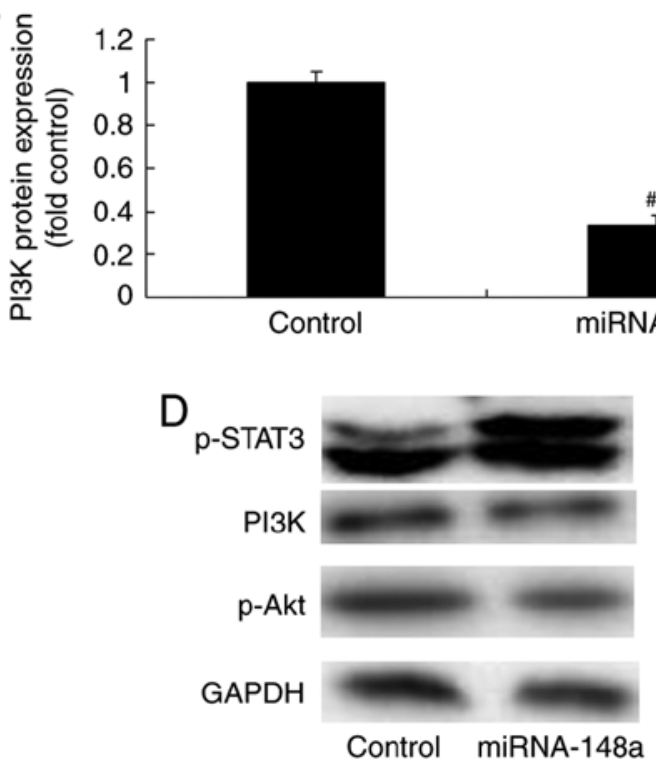

Figure 5. miRNA-148a overexpression suppresses p-STAT3, PI3K and p-Akt protein expression of PTC cells. miRNA-148a overexpression suppressed p-STAT3, PI3K and p-Akt protein expression by statistical analysis (A-C) and p-STAT3, PI3K and p-Akt protein expression by western blot analysis (D) of PTC cells. Control, control group; miRNA-148a, miRNA-148a overexpression group. ${ }^{\# \#} \mathrm{p}<0.01$ compared with control group.

miRNA-148a overexpression suppresses $p$-STAT3, PI3K and p-Akt protein expression of PTC cells. Western blot analysis was used to measure p-STAT3, PI3K and p-Akt protein expression of PTC cells after miRNA-148a overexpression. As shown in Fig. 5, miRNA-148a overexpression suppressed p-STAT3, PI3K and p-Akt protein expression of PTC cells.

si-STAT3 inhibits $p$-STAT3 protein expression, decreased cell proliferation and metastases of PTC cells after miRNA-148a overexpression. Then, we downregulated p-STAT3 protein expression in PTC cells following miRNA-148a overexpression. As shown in Fig. 6, si-STAT3 inhibits p-STAT3 protein expression in PTC cells following miRNA-148a overexpression, and decreased cell proliferation and metastases of
TPC-1 cells, compared with miRNA-148a overexpression group.

si-STAT3 induces apoptosis of PTC cells after miRNA-148a overexpression. Then, we found that apoptosis rate of TPC-1 cells after miRNA-148a overexpression in si-STAT3 were higher than that of miRNA-148a overexpression group (Fig. 7).

si-STAT3 induces Bax protein expression and caspase-3/9 levels of PTC cells after miRNA-148a overexpression. As expected, Bax protein expression and caspase-3/9 levels of PTC cells after miRNA-148a overexpression were markedly induced by si-STAT3, compared miRNA-148a overexpression 


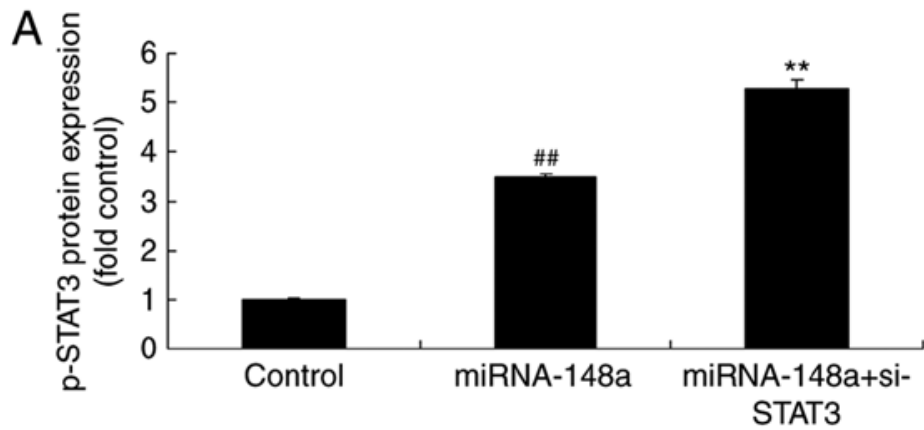

B

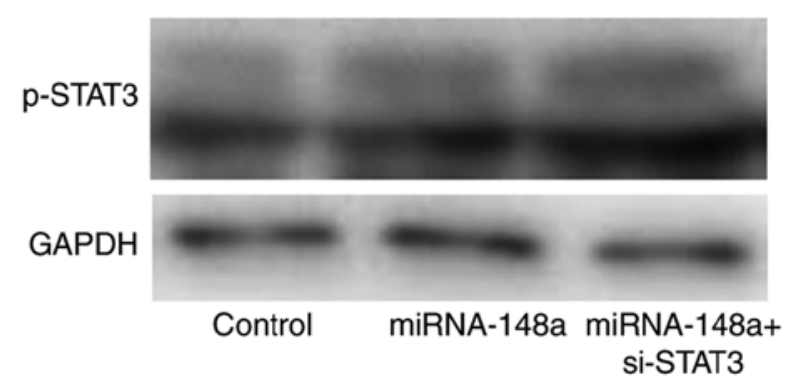

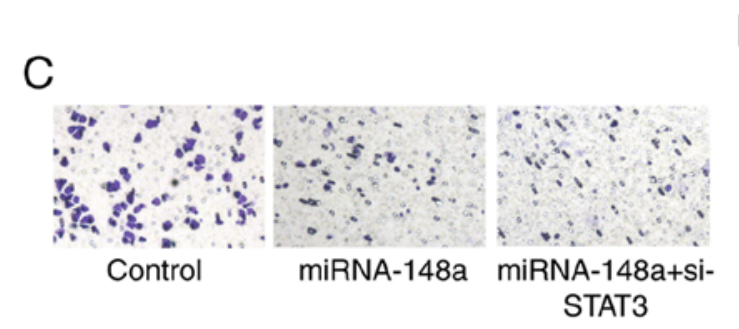

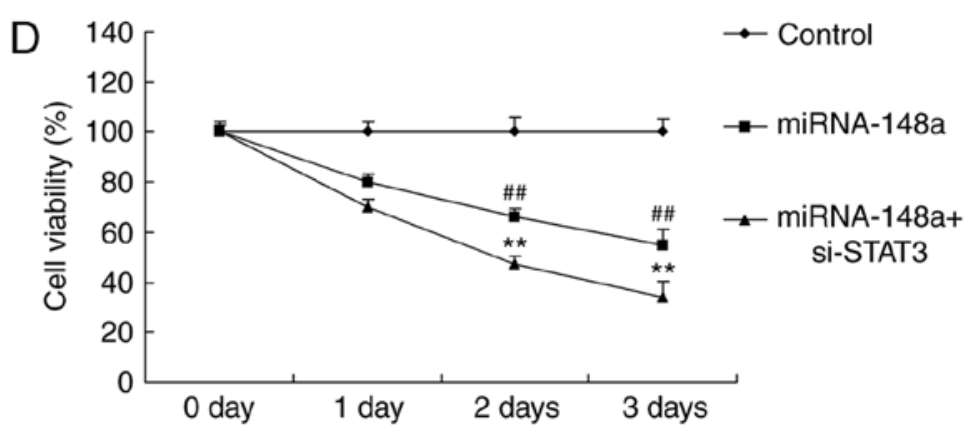

Figure 6. si-STAT3 inhibits p-STAT3 protein expression, reduced cell proliferation and metastases of PTC cells after miRNA-148a overexpression. p-STAT3 protein expression by statistical analysis (A) and p-STAT3 protein expression by western blot analysis (B), metastases (C) and cell proliferation (D) of PTC cells. Control, control group; miRNA-148a, miRNA-148a overexpression group; miRNA-148a+si-STAT3, miRNA-148a overexpression + si-STAT3 group. ${ }^{\#} \mathrm{p}<0.01$ compared with control group; ${ }^{* *} \mathrm{p}<0.01$ compared with miRNA-148a overexpression group.
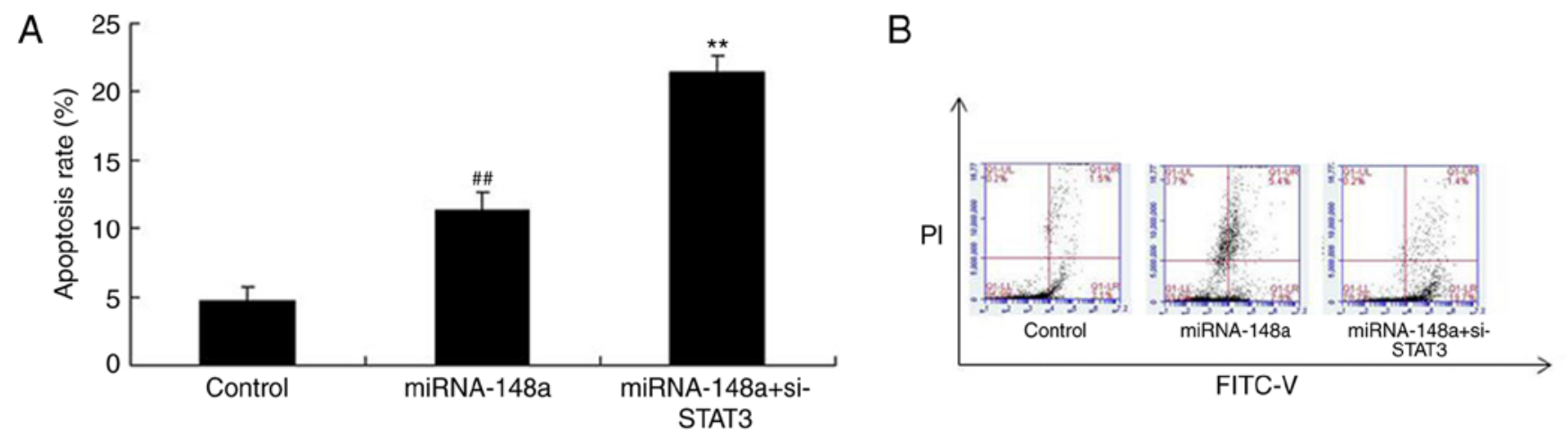

Figure 7. si-STAT3 increased apoptosis of PTC cells after miRNA-148a overexpression. si-STAT3 increased apoptosis rate (A) and cell apoptosis by flow cytometry (B). Control, control group; miRNA-148a, miRNA-148a overexpression group; miRNA-148a+si-STAT3, miRNA-148a overexpression + si-STAT3 group. ${ }^{\# \#} \mathrm{p}<0.01$ compared with control group; ${ }^{* *} \mathrm{p}<0.01$ compared with miRNA-148a overexpression group.

group (Fig. 8). From these results, we concluded that miR-148a functions as a suppressor in cell growth of PTC cells through inhibition of STAT3 expression.

PI3K inhibitor, inhibits PI3K and $p$-Akt protein expression of PTC cells after miRNA-148a overexpression. We next examined whether the inhibition of PI3K enforced miR-148a expression could suppress tumor growth of PTC. The results showed that PI3K inhibitor inhibits PI3K and p-Akt protein expression of PTC cells after miRNA-148a overexpression, compared with miRNA-148a overexpression group (Fig. 9).

PI3K inhibitor reduces cell proliferation and decreased metastases of PTC cells after miRNA-148a overexpression. Then, PI3K inhibitor increased the anticancer effects of
miRNA-148a overexpression on the inhibition of cell proliferation and metastases of PTC cells, compared with miRNA-148a overexpression group (Fig. 10).

PI3K inhibitor induces apoptosis of PTC cells after miRNA-148a overexpression. We determined whether PI3K inhibitor affects apoptosis of PTC cells after miRNA-148a overexpression. As shown in Fig. 11, PI3K inhibitor induced apoptosis of PTC cells after miRNA-148a overexpression, compared with miRNA-148a overexpression group (Fig. 11).

PI3K inhibitor induces Baxprotein expression and caspase-3/9 levels of PTC cells after miRNA-148a overexpression. Lastly, we found that PI3K inhibitor promoted the anticancer effects of miRNA-148a overexpression on the induction of Bax protein 

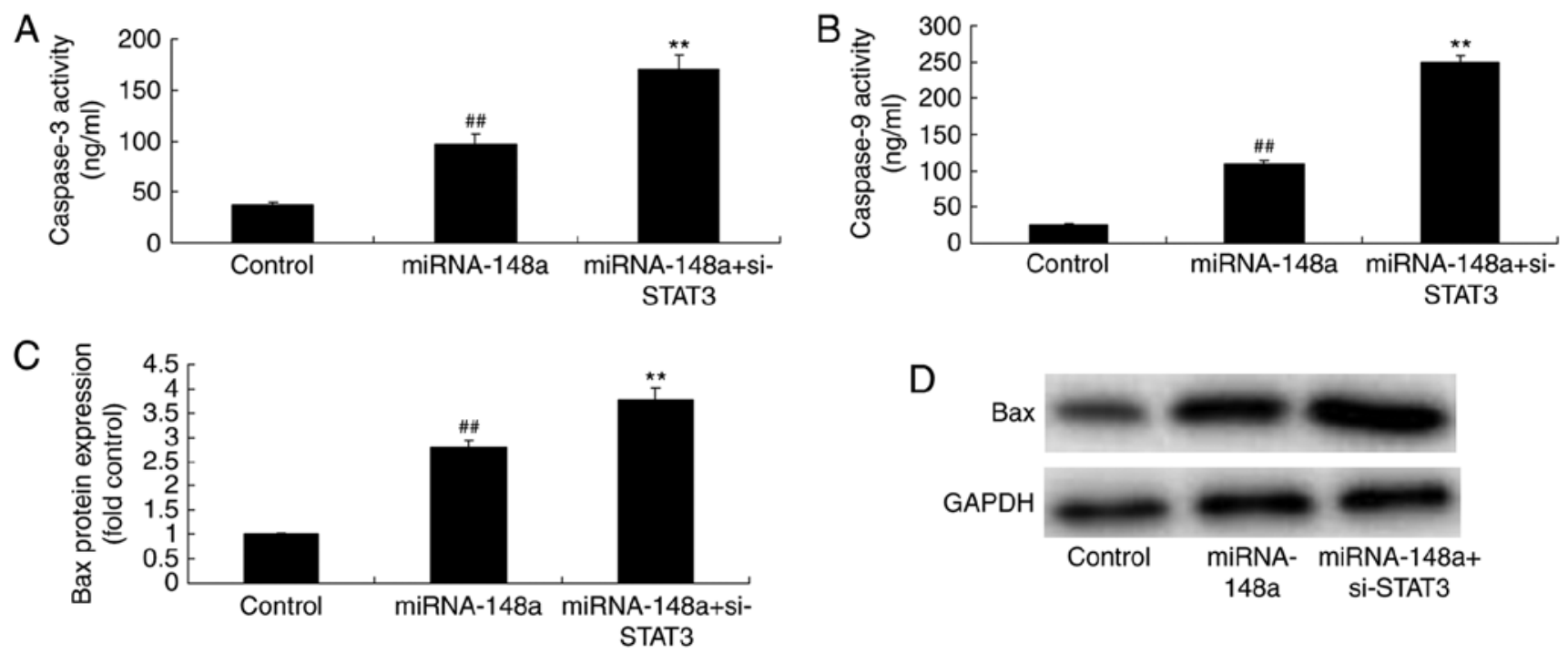

Figure 8. si-STAT3 induces Bax protein expression and caspase-3/9 levels of PTC cells after miRNA-148a overexpression. si-STAT3 induced caspase-3/9 levels (A and B), Bax protein expression by statistical analysis (C) and Bax protein expression by western blot analysis (D) of PTC cells. Control, control group; miRNA-148a, miRNA-148a overexpression group; miRNA-148a+si-STAT3, miRNA-148a overexpression + si-STAT3 group. ${ }^{\# \#}$ p $<0.01$ compared with control group; ${ }^{* *} \mathrm{p}<0.01$ compared with miRNA-148a overexpression group.
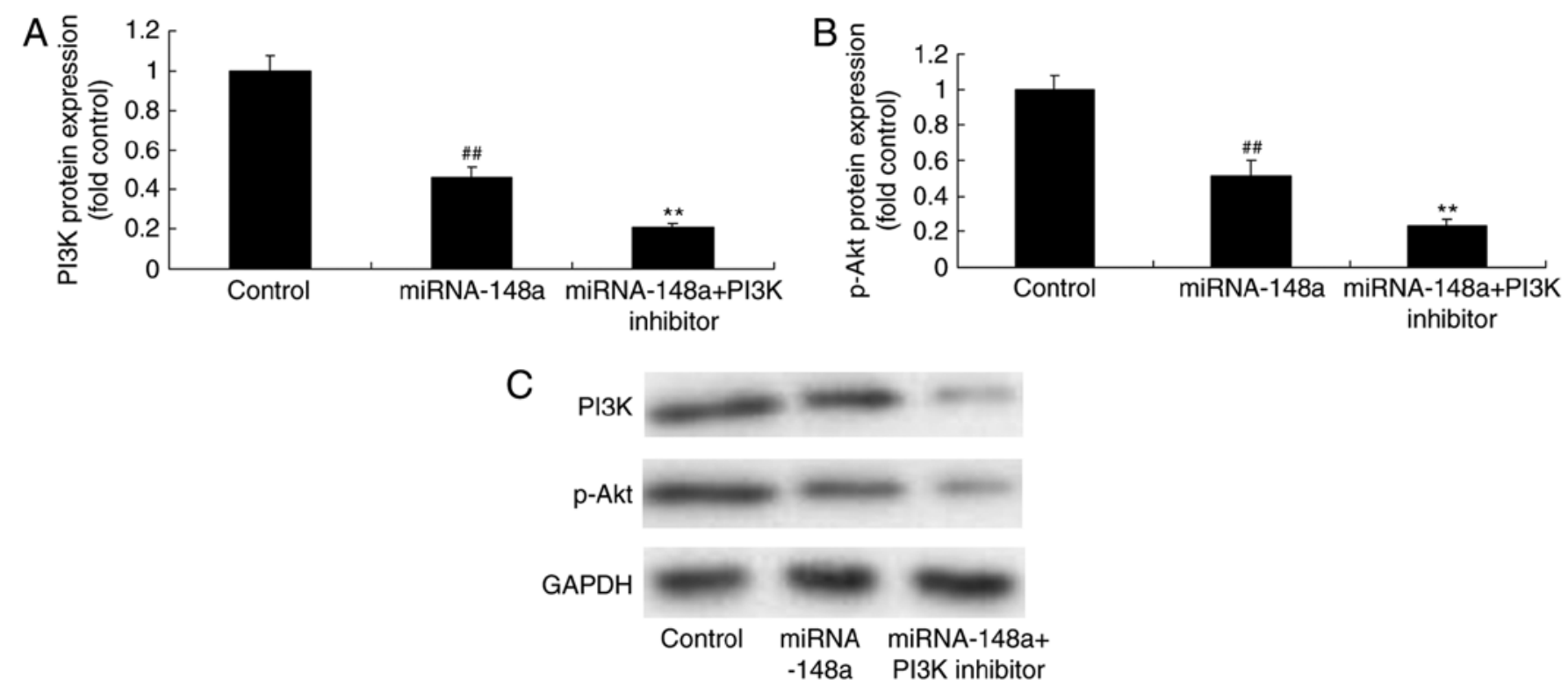

Figure 9. PI3K inhibitor, inhibits PI3K and p-Akt protein expression of PTC cells after miRNA-148a overexpression. PI3K and p-Akt protein expression by statistical analysis (A and B) and p-STAT3, PI3K and p-Akt protein expression by western blot analysis (C) of PTC cells. Control, control group; miRNA-148a, miRNA-148a overexpression group; miRNA-148a+ PI3K inhibitor, miRNA-148a overexpression + si-STAT3 group. ${ }^{* \# p<0.01 ~ c o m p a r e d ~ w i t h ~ c o n t r o l ~ g r o u p ; ~}$ *** $\mathrm{p}<0.01$ compared with miRNA-148a overexpression group.

expression and caspase-3/9 levels of PTC cells, compared with miRNA-148a overexpression group (Fig. 12).

\section{Discussion}

PTC is the most commonly seen malignant endocrine system tumor, which severely impair female health (16). The morbidity of PTC in China shows a significantly increasing trend in recent years, which is even higher in costal cities, exceeding the growth rate of other malignant tumors (17). Searching for new methods for the diagnosis and treatment of PTC is of particular importance since cancer has caused tremendous burdens on the society, families and individuals (18). PTC is a common human adenocarcinoma, which is dominated by papillary carcinoma, accounting for $\sim 70 \%$ of all PTCs (17). The growth rate of such cancer is relatively slow, with relatively low malignancy grade. However, it is adjacent to human neck and is prone to develop cervical lymph node metastasis (1). Our results showed that, for the first time, miRNA-148a expression of lymphatic metastases of papillary thyroid cancer patients was inhibited, compared with normal group. Feng et al showed that microRNA-148a suppressed cell proliferation and migration of pancreatic cancer cells (6). 
A

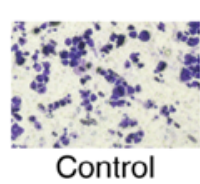

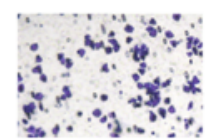

miRNA-148a

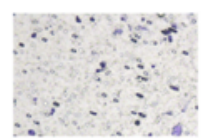

miRNA-148a+ PI3K inhibitor
B

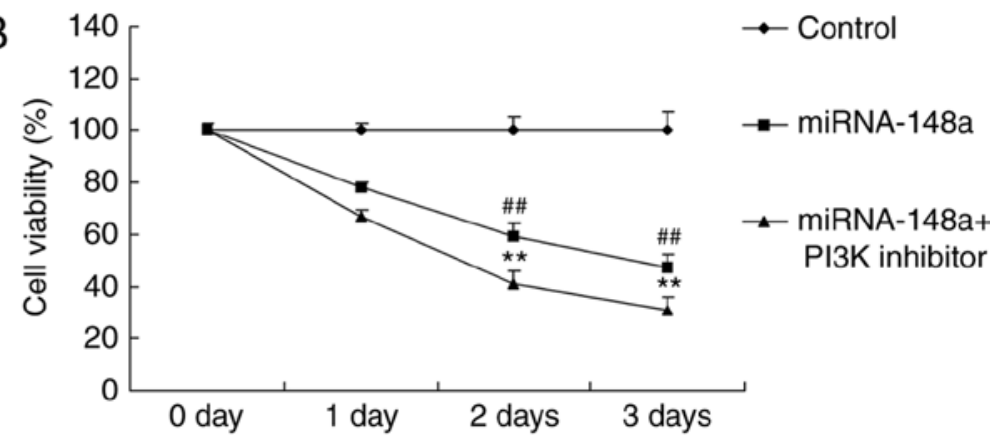

Figure 10. PI3K inhibitor reduces cell proliferation and induces metastases of PTC cells after miRNA-148a overexpression. inhibitor reduced metastases (A) and cell proliferation (B) of PTC cells. Control, control group; miRNA-148a, miRNA-148a overexpression group; miRNA-148a+ PI3K inhibitor, miRNA-148a overexpression + si-STAT3 group. ${ }^{\# \#} \mathrm{p}<0.01$ compared with control group; ${ }^{* *} \mathrm{p}<0.01$ compared with miRNA-148a overexpression group.
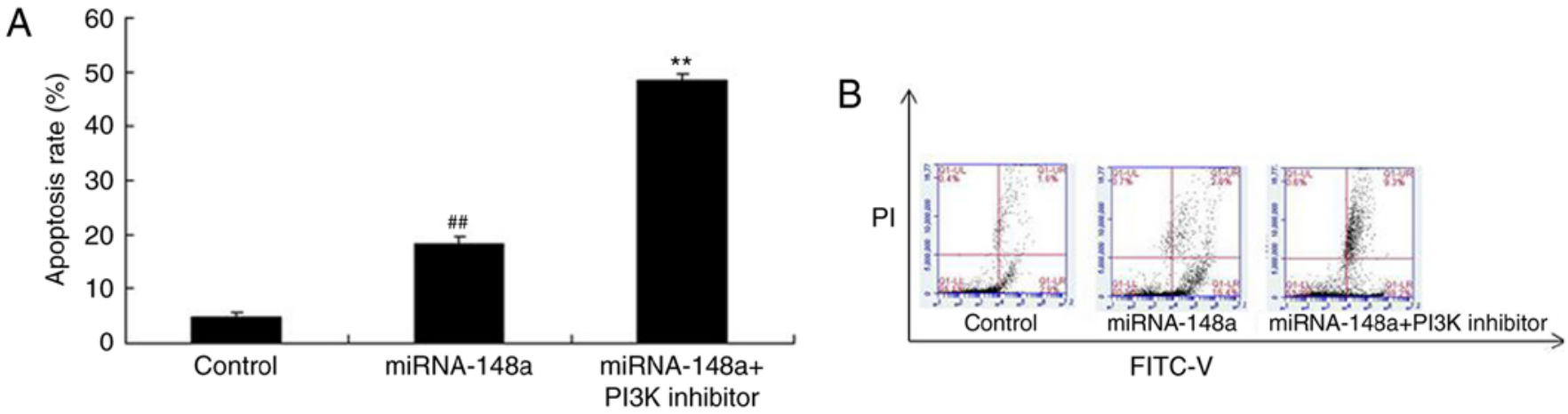

FITC-V

Figure 11. PI3K inhibitor increased apoptosis of PTC cells after miRNA-148a overexpression. PI3K inhibitor increased apoptosis rate (A) and cell apoptosis by flow cytometry (B). Control, control group; miRNA-148a, miRNA-148a overexpression group; miRNA-148a+PI3K inhibitor, miRNA-148a overexpression + si-STAT3 group. ${ }^{\# \#} \mathrm{p}<0.01$ compared with control group; ${ }^{* *} \mathrm{p}<0.01$ compared with miRNA-148a overexpression group.

$$
\text { A }
$$

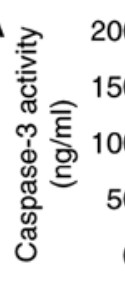
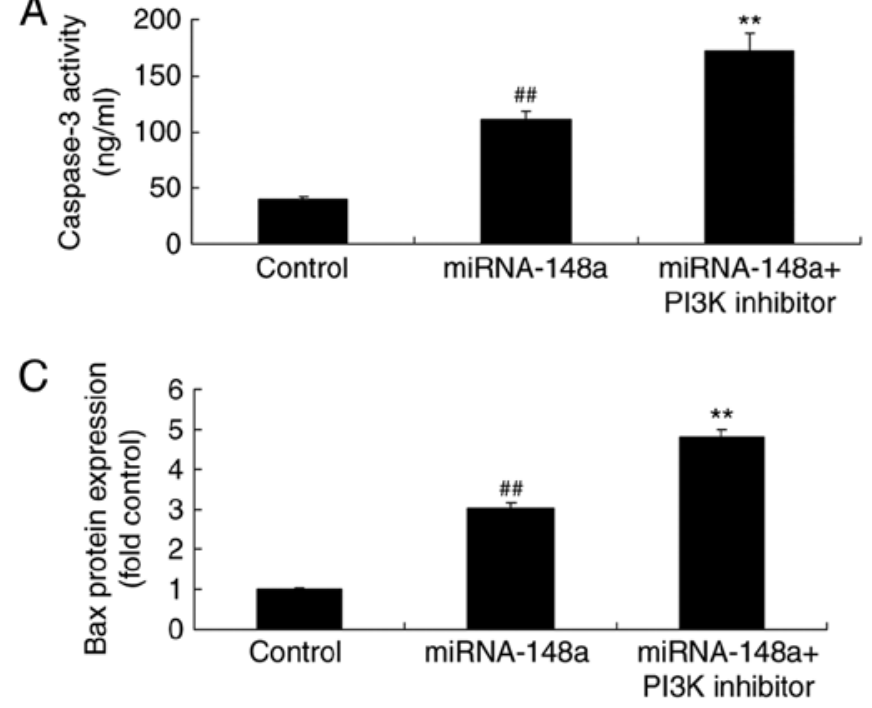
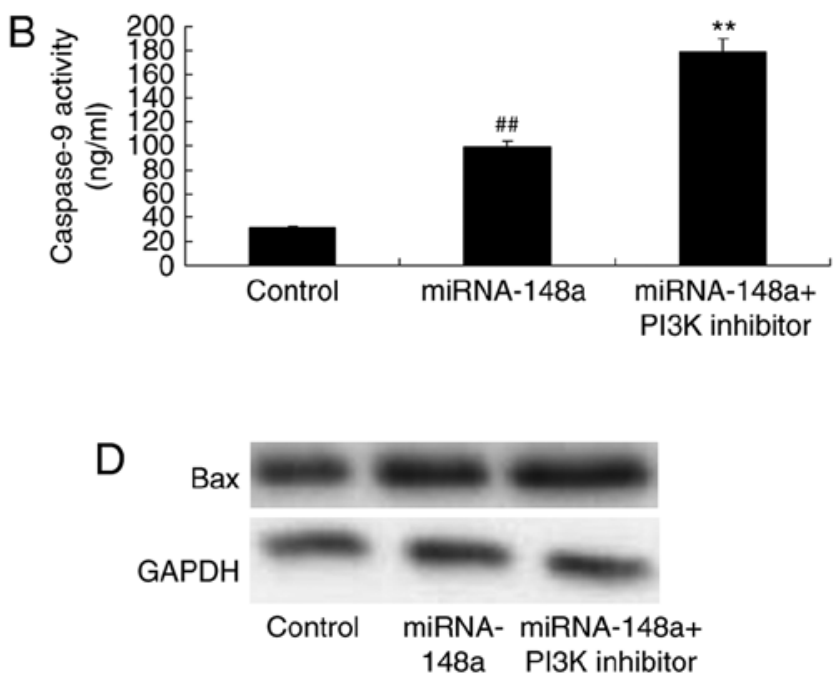

Figure 12. PI3K inhibitor induced Bax protein expression and caspase-3/9 levels of PTC cells after miRNA-148a overexpression. PI3K inhibitor induced caspase-3/9 levels (A and B), Bax protein expression by statistical analysis (C) and Bax protein expression by western blot analysis (D) of PTC cells. Control, control group; miRNA-148a, miRNA-148a overexpression group; miRNA-148a+ PI3K inhibitor, miRNA-148a overexpression + si-STAT3 group. ${ }^{\# \#}$ p $<0.01$ compared with control group; ${ }^{* *} \mathrm{p}<0.01$ compared with miRNA-148a overexpression group.

So far, $>700$ miRNAs in human genome have been identified (19). These microRNAs are the non-coding sequences in the genome with the length of $21-25$ nucleotides. They are highly conserved in evolution and can regulate gene expression at translation level (19), they participle in multiple physiological metabolic processes, including development, cell differentiation, apoptosis, and energy metabolism. With the advancement in research on molecular biology and molecular 
genetics, increasing number of studies have demonstrated that miRNAs play important roles in tumor development, metastasis and prognosis. For instance, miRNAs play certain roles in granulocytic leukemia, non-small cell lung cancer, gastric cancer, pancreatic cancer, colon cancer and liver cancer (20). Our results suggested that miRNA-148a overexpression effectively reduced cell-cell proliferation and metastases, and induced apoptosis of papillary thyroid cancer in vitro. Yu et al showed that miR-148a suppressed human gastric cancer cell growth by inactivating STAT3 and Akt (21). Li et al reported that silencing of miRNA-148a activates nasopharyngeal carcinoma, and can act as as a biomarker as well as a therapeutic target for nasopharyngeal carcinoma (22). These results were in keeping with our study.

Caspase family includes important proteins for executing apoptosis discovered in research on the molecular mechanism of cell apoptosis in recent years. Among them, caspase-3/9 is one of the pro-apoptotic factors locating in the upstream of caspase cascade reaction. Moreover, it is also one of the most critical components in the caspase system that induces cell apoptosis $(23,24)$. We also confirmed that overexpression of miRNA-148a significantly induced Bax protein expression and caspase-3/9 levels, and suppressed phosphorylation of STAT3 (p-STAT3), PI3K and p-Akt protein expression of papillary thyroid cancer in vitro.

STAT3 phosphorylation can result from multiple factors that promote the genesis and development of cancer. The activated STAT3 can inhibit cell apoptosis, promotes cell proliferation and differentiation, and results in carcinogenesis. Therefore, it is defined as an oncogene (25). Abnormally active STAT3 expression in PTC cells has been reported in relevant literature. Its expression difference is positively correlated with the clinical pathological type and stage of PTC (11). We showed that overexpression of miRNA-148a significantly suppressed $\mathrm{p}$-STAT3 protein expression of papillary thyroid cancer in vitro. si-STAT3, could inhibit p-STAT3 protein expression, reduced cell cell proliferation and metastases, and induced apoptosis of papillary thyroid cancer following miRNA-148a overexpression. He et al showed that microRNA-148a suppressed cell proliferation and invasion potential through regulation of STAT3 in non-small cell lung carcinomas (7).

The growth factor receptor, PI3K, Akt and eIF-4E in the PI3K/Akt/mTOR signaling pathway are all proteins encoded by oncogenes (26). It has been discovered currently that numerous tumors are accompanied with abnormal regulation of the mTOR signaling pathway. In addition, cell proliferation, cell cycle regulation, and cell migration, which are closely associated with tumor genesis, are all regulated by mTOR (13). It has been indicated in research that, the PI3K/ $\mathrm{Akt} / \mathrm{mTOR}$ signaling pathway is excessively activated in the genesis and development of PTC (15). The PI3K/Akt/mTOR signaling pathway is activated in PTC, especially in patients with lymph node metastases (27). It is proposed that the $\mathrm{PI} 3 \mathrm{~K} / \mathrm{Akt} / \mathrm{mTOR}$ signaling pathway supports the malignant characteristics of PTC cell model, and the effects of mTOR targeted therapy on advanced PTC have been approved (27). In our study, we determined that overexpression of miRNA-148a significantly suppressed PI3K and p-Akt of papillary thyroid cancer in vitro. PI3K inhibitor, could inhibit PI3K and $\mathrm{p}-\mathrm{Akt}$ protein expression, reduced cell cell proliferation and metastases, and induced apoptosis of papillary thyroid cancer following miRNA-148a overexpression. Zhang et al reported that microRNA-148a promotes cancer cell growth by targeting PI3K signaling pathway in osteosarcoma (7).

In conclusion, our results indicate that miRNA-148a inhibits cell growth and metastases of papillary thyroid cancer through STAT3 and PI3K/AKT signaling pathways. To our knowledge, this is the first study to describe a potential role for miRNA-148a as potential targets in future studies on the prevention and treatment of papillary thyroid cancer.

\section{References}

1. Sawka AM, Straus S, Rodin G, Thorpe KE, Ezzat S, Gafni A and Goldstein DP: Decision aid on radioactive iodine treatment for early stage papillary thyroid cancer: Update to study protocol with follow-up extension. Trials 16: 302, 2015.

2. Yuan Y, Fang M, Wu CY and Ling EA: Scutellarin as a potential therapeutic agent for microglia-mediated neuroinflammation in cerebral ischemia. Neuromolecular Med 18: 264-273, 2016.

3. Titov SE, Ivanov MK, Karpinskaya EV, Tsivlikova EV, Shevchenko SP, Veryaskina YA, Akhmerova LG, Poloz TL, Klimova OA, Gulyaeva LF, et al: miRNA profiling, detection of BRAF V600E mutation and RET-PTC1 translocation in patients from Novosibirsk oblast (Russia) with different types of thyroid tumors. BMC Cancer 16: 201, 2016.

4. Yoruker EE, Terzioglu D, Teksoz S, Uslu FE, Gezer U and Dalay N: MicroRNA expression profiles in papillary thyroid carcinoma, benign thyroid nodules and healthy controls. J Cancer 7: 803-809, 2016.

5. Mutalib NS, Yusof AM, Mokhtar NM, Harun R, Muhammad R and Jamal R: MicroRNAs and lymph node metastasis in papillary thyroid cancers. Asian Pac J Cancer Prev 17: 25-35, 2016.

6. Feng H, Wang Y, Su J, Liang H, Zhang CY, Chen X and Yao W: MicroRNA-148a suppresses the proliferation and migration of pancreatic cancer cells by downregulating ErbB3. Pancreas 45: 1263-1271, 2016.

7. Zhang H, Wang Y, Xu T, Li C, Wu J, He Q, Wang G, Ding C, Liu K, Tang H, et al: Increased expression of microRNA-148a in osteosarcoma promotes cancer cell growth by targeting PTEN. Oncol Lett 12: 3208-3214, 2016.

8. Zhang J, Gill A, Atmore B, Johns A, Delbridge L, Lai R and McMullen T: Upregulation of the signal transducers and activators of transcription 3 (STAT3) pathway in lymphatic metastases of papillary thyroid cancer. Int J Clin Exp Pathol 4: 356-362, 2011.

9. Kim YR, Byun HS, Won M, Park KA, Kim JM, Choi BL, Lee H, Hong JH, Park J, Seok JH, et al: Modulatory role of phospholipase D in the activation of signal transducer and activator of transcription (STAT)-3 by thyroid oncogenic kinase RET/PTC. BMC Cancer 8: 144, 2008.

10. Kim DW, Chung HK, Park KC, Hwang JH, Jo YS, Chung J, Kalvakolanu DV, Resta $\mathrm{N}$ and Shong M: Tumor suppressor LKB1 inhibits activation of signal transducer and activator of transcription 3 (STAT3) by thyroid oncogenic tyrosine kinase rearranged in transformation (RET)/papillary thyroid carcinoma (PTC). Mol Endocrinol 21: 3039-3049, 2007.

11. Couto JP, Daly L, Almeida A, Knauf JA, Fagin JA, SobrinhoSimões M, Lima J, Máximo V, Soares P, Lyden D, et al: STAT3 negatively regulates thyroid tumorigenesis. Proc Natl Acad Sci USA 109: E2361-E2370, 2012.

12. Trovato M, Grosso M, Vitarelli E, Ruggeri RM, Alesci S, Trimarchi F, Barresi G and Benvenga S: Distinctive expression of STAT3 in papillary thyroid carcinomas and a subset of follicular adenomas. Histol Histopathol 18: 393-399, 2003.

13. Liu Q, Guan JZ, Sun Y, Le Z, Zhang P, Yu D and Liu Y: Insulin-like growth factor 1 receptor-mediated cell survival in hypoxia depends on the promotion of autophagy via suppression of the PI3K/Akt/mTOR signaling pathway. Mol Med Rep 15: 2136-2142, 2017.

14. Mao Y, Xi L, Li Q, Cai Z, Lai Y, Zhang X and Yu C: Regulation of cell apoptosis and proliferation in pancreatic cancer through PI3K/Akt pathway via Polo-like kinase 1. Oncol Rep 36: 49-56, 2016. 
15. Petrulea MS, Plantinga TS, Smit JW, Georgescu CE and Netea-Maier RT: PI3K/Akt/mTOR: A promising therapeutic target for non-medullary thyroid carcinoma. Cancer Treat Rev 41: 707-713, 2015.

16. Gweon HM, Son EJ, Youk JH, Kim JA and Park CS: Preoperative assessment of extrathyroidal extension of papillary thyroid carcinoma: Comparison of 2- and 3-dimensional sonography. J Ultrasound Med 33: 819-825, 2014.

17. Lei S, Wang D, Ge J, Liu H, Zhao D, Li G and Ding Z: Singlecenter study of familial papillary thyroid cancer in China: Surgical considerations. World J Surg Oncol 13: 115, 2015.

18. Proczko M, Stefaniak T, Sworczak K, Kobiela J, Lachiński AJ, Stepaniak P and Sledziński Z: Completion thyroidectomy of well-differentiated thyroid cancer - a prospective, randomised study. Endokrynol Pol 64: 335-339, 2013.

19. Shen CT, Qiu ZL, Song HJ, Wei WJ and Luo QY: miRNA-106a directly targeting RARB associates with the expression of $\mathrm{Na}(+) / \mathrm{I}(-)$ symporter in thyroid cancer by regulating MAPK signaling pathway. J Exp Clin Cancer Res 35: 101, 2016.

20. Geraldo MV, Nakaya HI and Kimura ET: Downregulation of 14q32-encoded miRNAs and tumor suppressor role for miR-654-3p in papillary thyroid cancer. Oncotarget 8: 9597-9607, 2017.

21. Yu B, Lv X, Su L, Li J, Yu Y, Gu Q, Yan M, Zhu Z and Liu B: MiR-148 a functions as a tumor suppressor by targeting CCK-BR via inactivating STAT3 and Akt in human gastric cancer. PLoS One 11: e0158961, 2016.
22. Li HP, Huang HY, Lai YR, Huang JX, Chang KP, Hsueh C and Chang YS: Silencing of miRNA-148a by hypermethylation activates the integrin-mediated signaling pathway in nasopharyngeal carcinoma. Oncotarget 5: 7610-7624, 2014.

23. Jin SM, Jang HW, Sohn SY, Kim NK, Joung JY, Cho YY, Kim SW and Chung JH: Role of autophagy in the resistance to tumour necrosis factor-related apoptosis-inducing ligandinduced apoptosis in papillary and anaplastic thyroid cancer cells. Endocrine 45: 256-262, 2014.

24. Saffar H, Sanii S, Emami B, Heshmat R, Panah VH, Azimi S and Tavangar SM: Evaluation of MMP2 and Caspase-3 expression in 107 cases of papillary thyroid carcinoma and its association with prognostic factors. Pathol Res Pract 209: 195-199, 2013.

25. Dong W, Cui J, Tian X, He L, Wang Z, Zhang P and Zhang H: Aberrant sonic hedgehog signaling pathway and STAT3 activation in papillary thyroid cancer. Int J Clin Exp Med 7: 1786-1793, 2014.

26. Zhao P, Guan HT, Dai ZJ, Ma YG, Liu XX and Wang XJ: Knockdown of SPOCK1 inhibits the proliferation and invasion in colorectal cancer cells by suppressing the PI3K/Akt pathway. Oncol Res 24: 437-445, 2016.

27. Gonçalves AP, Videira A, Soares P and Máximo V: Orthovanadateinduced cell death in RET/PTC1-harboring cancer cells involves the activation of caspases and altered signaling through PI3K/ Akt/mTOR. Life Sci 89: 371-377, 2011. 